\title{
A safety mechanism for observational learning
}

\author{
Arnaud Badets $^{1}$ - Arnaud Boutin ${ }^{2,3} \cdot$ Thomas Michelet $^{1,4,5}$
}

Published online: 27 July 2017

(C) Psychonomic Society, Inc. 2017

\begin{abstract}
This empirical article presents the first evidence of a "safety mechanism" based on an observational-learning paradigm. It is accepted that during observational learning, a person can use different strategies to learn a motor skill, but it is unknown whether the learner is able to circumvent the encoding of an uncompleted observed skill. In this study, participants were tested in a dyadic protocol in which an observer watched a participant practicing two different motor sequences during a learning phase. During this phase, one of the two motor sequences was interrupted by a stop signal that precluded motor learning. The results of the subsequent retention test revealed that both groups learned the two motor sequences, but only the physical practice group showed worse performance for the interrupted sequence. The observers were consequently able to use a safety strategy to learn both sequences equally. Our findings are discussed in light of the implications of the action observation network for sequence learning and the cognitive mechanisms of error-based observation.
\end{abstract}

Arnaud Badets and Arnaud Boutin contributed equally to this work.

Arnaud Badets

arnaud.badets@u-bordeaux.fr

1 CNRS, Institut de Neurosciences Cognitives et Intégratives d'Aquitaine (UMR 5287), Université de Bordeaux, Bordeaux, France

2 Unité de Neuroimagerie Fonctionnelle, C.R.I.U.G.M., Montréal, QC, Canada

3 Université de Montréal, Montréal, QC, Canada

4 Institut des Maladies Neurodégénératives, Université de Bordeaux, Bordeaux, France

5 Institut des Maladies Neurodégénératives, CNRS, Bordeaux, France
Keywords Motor sequence - Observational learning · Learning strategy

Observational learning represents a crucial adaptation strategy in different real-life situations. Indeed, an observer does not physically practice the task and consequently he or she does not risk injury or expend the same amount of energy as a practitioner during learning. For example, the recovery of upper limb function in patients following stroke is enhanced after action observation (Franceschini et al., 2010). In sports situations or surgical performance, observation is a useful tool to convey information to learners (Hodges, Williams, Hayes, \& Breslin, 2007). All these findings emphasize the fact that at a neurophysiological level, common neuronal network activation is shared between physical and observational practice, known as the action observation network (AON), which includes the premotor cortex, the primary motor cortex, and the inferior parietal cortex (Press, 2011). From the AON perspective, it could be hypothesized that all watched skills can engage at some level the representation of an action in observers. Consequently, it could be argued that if an observer watches an interrupted skill, the interruption will be processed and, in turn, will decrease motor performance during subsequent enactment. In contrast, if observers were able to learn a task without being impacted by the practitioner's interruption, we could argue that any observed perturbation could be avoided during observational learning. This present article presents the first evidence for this "safety" hypothesis.

Theoretically, the safety hypothesis can accord with the concept of emulation, which emphasizes the ability in humans to learn from the goals or intentions of others, whatever the specific sequential actions taken to attain these goals (Behne, Carpenter, Call, \& Tomasello, 2005; Meltzoff, 1995; Subiaul, Patterson, \& Barr, 2016). In this view, an interrupted or a 
noninterrupted task can similarly afford the goal of the to-beenacted action. Accordingly, for observational learning, this goal emulation could imply learning from the model's intended goal but not from the model's movement (Loucks \& Meltzoff, 2013; Whiten \& Ham, 1992). In the same vein, some behavioral studies have revealed that physical and observational practice could rely on specific cognitive mechanisms (Badets \& Blandin, 2010; Badets, Blandin, Bouquet, \& Shea, 2006; Hayes, Elliott, Andrew, Roberts, \& Bennett, 2012; Shea, Wright, Wulf, \& Whitacre, 2000). For this dissociation between physical and observational practice, Thomas, Sink, and Haggard (2013) stated that "action observation may be dominated by simulation of the model's sensations, rather than their motor commands" (p. 344). Such a statement accords with previous studies showing that observational learning relies mainly on a visual-spatial representation (Boutin, Fries, Panzer, Shea, \& Blandin, 2010; Hayes et al., 2012).

Accordingly, Badets and Blandin (2010) proposed that an observational condition should engage participants on the perceptual (and not motor) level of the action to be learned (see also Maslovat, Hodges, Krigolson, \& Handy, 2010, and Buchanan, 2015, for limitations of this statement in coordination tasks). Empirical evidence for such a claim comes from the delayed motor intention (DMI) paradigm. A delayed motor intention is the capacity to imagine in a far future an action to be performed. Several domains, such as skill learning, tool use, or action memory, revealed that this foresight capacity engages mainly a perceptual mechanism for the practitioner (see Badets \& Osiurak, 2015, for a review and the theory). For example, Badets et al. (2006, Exp. 3) have found that, in contrast to physical practice conditions, an observational practice condition benefited from this DMI. In this study, participants were required to learn four different motor sequences during an encoding phase based on a physical or an observational practice condition. Before this encoding phase, two motor sequences were associated with a DMI (i.e., the instruction emphasized that participants will have to reproduce the two motor sequences during a future retention phase), and two motor sequences were not associated with a DMI. The retention phase was performed after the encoding phase. The results of the observational group during the retention phase revealed that learning of the two motor sequences associated with a DMI was improved as compared to the two motor sequences not associated with a DMI. For the physical practice condition, the motor representation (and to a lesser extent, the perceptual level) is mainly involved during the production of the to-be learned movement, and as a result the DMI cannot improve the skill level through this enactment.

Another interesting specificity of observational learning is the capacity to implement different strategies to efficiently learn the task. For example, it is accepted that observing both an expert and a novice model improves skill learning as compared to the mere observation of either an expert or a novice model alone (Andrieux \& Proteau, 2013; Rohbanfard $\&$ Proteau, 2011). For Andrieux and Proteau, such a mixed observational-learning procedure provides the learner an accurate reference of the skill to be performed from an expert model, and typical errors from the novice give the learner the opportunity to avoid them during encoding (see also Badets et al., 2006). This last interpretation accords with a safety mechanism for observers. However, it is unknown whether a sudden interruption of the task to be learned can be avoided by an observer. Specifically, during physical practice learning, it could be hypothesized that an interruption during the encoding phase should weaken the learning process of the task. From the AON viewpoint, the weakening of acquisition performance should be equally exhibited during a retention phase for the physical practice group and for the observational practice group. In contrast, if an observer can avoid detected errors during observational learning, then such interruption should not impede skill learning (Andrieux \& Proteau, 2014; Badets et al., 2006).

The following experiment tested these two alternatives hypotheses with a motor sequence-learning task. Participants were tested in a dyadic protocol during the acquisition phase, in which an observer learned the task from the actual performance of a learning model. The physical practice (PP) and observational (OBS) groups were required to learn two different motor sequences with their right hand (Motor Sequences $\mathrm{A}$ and $\mathrm{B}$ ), and they were instructed that during the acquisition phase only Motor Sequence B would be interrupted. To assess the learning level of the two motor sequences between groups, all participants performed different recall tests - that is, a retention test and two intermanual (visual-spatial and motor) transfer tests. The retention test was performed with the right hand and assessed the motor and visual-spatial components of the task. The motor-transfer test was performed with the left hand, and assessed the motor representation while keeping constant the order of musculoskeletal constraints of the task. The visual-spatial transfer test was performed with the left hand, and assessed the visual-spatial representation while keeping constant the order of target constraints of the task (see Boutin et al., 2010).

\section{Method}

\section{Participants}

Twenty-eight right-handed ( $N=14$ in each group) participants (mean age $\mathrm{PP}=18.6, S D=1.1$ [12 females]; mean age OBS = $18.5, S D=0.7$ [12 females]) from the University of Bordeaux were randomly assigned to two experimental groups (PP or OBS), which were defined according to the practice conditions they engaged in during the acquisition phase (Table 1 
Table 1 Experimental groups and procedures

\begin{tabular}{|c|c|c|c|c|c|c|c|c|}
\hline \multirow[b]{2}{*}{ Group } & \multicolumn{3}{|l|}{ Pretest } & \multirow[b]{2}{*}{ Acquisition } & \multicolumn{4}{|l|}{ Posttest } \\
\hline & $\mathrm{R}$ & VS & M & & $\mathrm{R}$ & VS & M & New \\
\hline PP & Seq $A / B$ & Seq $A / B$ & Mirrored Seq A/B & Seq $A / B$ & Seq A/B & Seq $A / B$ & Mirrored Seq A/B & Seq New \\
\hline OBS & Seq $A / B$ & Seq A/B & Mirrored Seq A/B & Seq A/B (Observation only) & Seq A/B & Seq $A / B$ & Mirrored Seq A/B & Seq New \\
\hline
\end{tabular}

All groups physically performed two motor-sequence tasks during the pretest and posttest phases. For the observational practice group (OBS), the acquisition session consisted of an observation phase, whereas for the physical practice group (PP), it consisted of physical practice. The acquisition and retention $(\mathrm{R})$ test sessions were performed with the dominant, right hand. The visual-spatial (VS) and motor (M) transfer tests were performed with the nondominant, left hand. A new sequence was presented during the posttest phase and was performed with the right hand.

summarizes the groups and experimental phases). All participants were unaware of the purpose of the study, were righthanded (Edinburgh Handedness Inventory; Oldfield, 1971), and had normal or corrected-to-normal vision. Instruction and informed consent were administered and recorded before the experiment.

\section{Apparatus, task, and procedure}

Participants sat on a chair in front of a computer screen, positioned in such a way that the viewing distance was approximately $50 \mathrm{~cm}$. Four horizontally aligned white empty squares $(2.1 \mathrm{~cm}$ wide $\times 2.1 \mathrm{~cm}$ high, spaced $1 \mathrm{~cm}$ apart $)$ were presented on a black background in the center of the screen and matched the spatial positions of the response keys $(\mathrm{C}, \mathrm{V}, \mathrm{B}$, and $\mathrm{N}$ ) on the AZERTY keyboard. Each imperative stimulus consisted in one of the four squares filled in blue and required participants to press the appropriate response key. Stimulus presentation and response registration were controlled using the MATLAB R2012b software from The MathWorks (Natick, MA) and the Psychophysics Toolbox extensions (Kleiner, Brainard, \& Pelli, 2007).

When physically practicing the task, participants were asked to respond as rapidly and accurately as possible to the sequentially presented stimuli by pressing the appropriate response keys on the keyboard. Each block of trials began with the presentation of the four horizontal empty squares accompanied by a brief 50-ms tone. The first imperative stimulus was presented after a foreperiod of variable duration (1-3 s in 0.5 -s steps). The response of the participant triggered the presentation of the next stimulus until completion of the practice block. Each practice block consisted of ten repetitions of a second-order conditional 12-element motor sequence (i.e., a total of 120 keypresses). At the end of each practice block, the display was erased and the screen remained black for $20 \mathrm{~s}$. When the participants were ready to proceed with the next practice block, they pressed any one of the four response keys.

The experimental procedure was composed of three main phases: pretest, acquisition, and posttest. In the pretest, all participants physically performed three different retention and intermanual (visual-spatial and motor) transfer tests.
The retention test was done first with the dominant hand. This test consisted of two practice blocks, with one block composed of Motor Sequence A (keys N-C-B-V-C-N-V$\mathrm{B}-\mathrm{N}-\mathrm{B}-\mathrm{C}-\mathrm{V}$ ) and one block of Motor Sequence B (keys $\mathrm{V}-$ $\mathrm{N}-\mathrm{V}-\mathrm{C}-\mathrm{B}-\mathrm{N}-\mathrm{C}-\mathrm{V}-\mathrm{B}-\mathrm{C}-\mathrm{N}-\mathrm{B})$. Both transfer tests were performed with the contralateral, nondominant left hand. In the motor test, the original Motor Sequences A and B were mirrored so that the sequential movements remained the same when transferred to the left hand (keys $\mathrm{C}-\mathrm{N}-\mathrm{V}-\mathrm{B}-\mathrm{N}-\mathrm{C}-\mathrm{B}-$ $\mathrm{V}-\mathrm{C}-\mathrm{V}-\mathrm{N}-\mathrm{B}$ and $\mathrm{B}-\mathrm{C}-\mathrm{B}-\mathrm{N}-\mathrm{V}-\mathrm{C}-\mathrm{N}-\mathrm{B}-\mathrm{V}-\mathrm{N}-\mathrm{C}-\mathrm{V}$ for Motor Sequences A and B, respectively). In the visual-spatial tests, the original Motor Sequences $\mathrm{A}$ and $\mathrm{B}$ were preserved but performed with the left hand (keys $\mathrm{N}-\mathrm{C}-\mathrm{B}-\mathrm{V}-\mathrm{C}-\mathrm{N}-\mathrm{V}-$ $\mathrm{B}-\mathrm{N}-\mathrm{B}-\mathrm{C}-\mathrm{V}$ and $\mathrm{V}-\mathrm{N}-\mathrm{V}-\mathrm{C}-\mathrm{B}-\mathrm{N}-\mathrm{C}-\mathrm{V}-\mathrm{B}-\mathrm{C}-\mathrm{N}-\mathrm{B}$ for Motor Sequences $\mathrm{A}$ and $\mathrm{B}$, respectively). Note that because of the present PP-OBS dyadic protocol, which involved the presence of the two PP and OBS counterparts in the room during the acquisition phase, we administered the pretest phase for each practice-group participant separately. Hence, while one participant was completing the pretest, his or her practice-group counterpart was waiting outside the testing place, and vice versa (order counterbalanced across participants).

The acquisition phase involved physical and observational practice conditions. Each participant in the OBS group was yoked to one participant in the PP group, such that the yoked counterpart received effective information from a learning model (Boutin et al., 2010). Participants in the PP group sat in front of the computer screen and physically performed 18 blocks of trials on Motor Sequences A and B with their dominant, right hand. Nine blocks were composed of Motor Sequence A, and nine blocks of Motor Sequence B. The practice of Motor Sequences A and B was alternated on a regular basis for every practice block across the acquisition session (e.g., Sequence A, Sequence B, Sequence A, etc.; order counterbalanced across participants). The participants in the OBS group stood upright and behind their PP group counterparts, located above the right shoulder to capture the visual information from the right-hand finger movements and successive stimuli on the screen. This observational viewing angle was adopted in order to closely match the perspective view 
of the participant actually performing the task. Where the practice of Motor Sequence A was entirely completed (noninterrupted) throughout the acquisition phase, the practice of Motor Sequence B was experimentally interrupted using a fading procedure, giving the PP and OBS participants the opportunity to learn the task during early practice; motortask interruption occurred with the visual presentation of the word "stop" in the center of the screen. More precisely, 100\% of trials were not interrupted for Blocks 1 and 2 (i.e., 120 keypresses completed), $75 \%$ of trials were not interrupted for Blocks 3 and 4 (i.e., 90 keypresses completed), 50\% of trials were not interrupted for Blocks 5 and 6 (i.e., 60 keypresses completed), and $25 \%$ of trials were not interrupted for Blocks 8 and 9 (i.e., 30 keypresses completed).

Approximately $5 \mathrm{~min}$ after completion of the acquisition phase, all participants underwent a posttest session. The procedure was identical to that of the pretest phase, except that participants performed an extra practice block on a new SOC motor sequence in order to differentiate motor-sequence learning from generalized or nonspecific practice effects. This new 12-element motor sequence (keys $\mathrm{V}-\mathrm{N}-\mathrm{C}-\mathrm{B}-\mathrm{V}-\mathrm{C}-\mathrm{N}-\mathrm{V}-\mathrm{B}-$ $\mathrm{N}-\mathrm{B}-\mathrm{C}$ ) was performed with the right hand.

\section{Results}

Response time (RT) was defined as the time interval between stimulus onset and the pressing of the corresponding key. Mean RT and error data were computed separately for each practice block during the pretest, acquisition, and posttest phases. Where significant effects were found $(p<.05)$, Duncan's multiple-range tests was used for post-hoc comparisons to determine the specific effects contributing to the general analysis of variance (ANOVA) effects. Partial eta-squared $\left(\eta_{\mathrm{p}}{ }^{2}\right)$ was the effect size reported for all significant effects (Cohen, 1988). Outliers $( \pm 2 S D)$ and the RTs from error trials (i.e., erroneous key presses) were excluded from the analyses (less than $5 \%$ in both groups).

\section{Speed: RT data analysis}

Pretest To assess basic skill levels and ensure that both groups were exhibiting similar performance on both sequence tasks during the pretest session, we ran a Group $(\mathrm{PP}, \mathrm{OBS}) \times$ Test (retention, visual-spatial transfer, motor transfer) $\times$ Sequence (A, B) ANOVA with repeated measures on the factors Test and Sequence. As expected, the analysis failed to detect a significant main effect of either group, $F(1,26)=1.66$, or sequence, $F(1,26)=1.40$, or a significant Group $\times$ Test, $F(2,52)<1$; Group $\times$ Sequence, $F(1,26)<1$; Test $\times$ Sequence, $F(2,52)<$ 1 ; or Group $\times$ Test $\times$ Sequence, $F(2,52)<1$, interaction, $p s>$ .05 . Only the main effect of test reached significance, $F(2,52)$ $=14.55, p<.001, \eta_{\mathrm{p}}{ }^{2}=.36$, with better performances on the retention test $(M=565 \mathrm{~ms})$ than on the intermanual visualspatial $(M=612 \mathrm{~ms} ; p<.001)$ and motor-transfer tests $(M=$ $610 \mathrm{~ms} ; p<.001)$, which did not differ $(p>.05)$. This effect reflected the switch from the dominant (i.e., retention test) to the nondominant (i.e., transfer tests) hand during motorsequence performance.

Acquisition Since the OBS group did not physically practice the task during the acquisition phase, this analysis was conducted with the data from the PP group only. Due to the interruption procedure during the acquisition of Motor Sequence B, we separately analyzed (i) the noninterrupted acquisition blocks during early practice, using a Block (1-2) $\times$ Sequence $(\mathrm{A}, \mathrm{B})$ ANOVA with repeated measures on both factors, and (ii) the following seven practice blocks, using a Block (3-9) $\times$ Sequence $(A, B)$ factorial design, again with repeated measures on both factors. (i) The first analysis revealed a block main effect, $F(1,13)=9.50, p<.01, \eta_{\mathrm{p}}{ }^{2}=$ .42 , with lower RTs on Block $1(M=474 \mathrm{~ms})$ than on Block 2 $(M=489 \mathrm{~ms} ; p<.01)$. The main effect of sequence, $F(1,13)=$ 2.40, and the Block $\times$ Sequence interaction, $F(1,13)<1$, were not significant, $p \mathrm{~s}>.05$. The results indicated similar skill levels on both motor sequences during early practice, with a slight but significant practice-dependent deterioration of task performance from Block 1 to Block 2 due to a potential interference effect from the interleaved practice of the two sequences. (ii) The second analysis revealed main effects of block, $F(6,78)=3.66, p<.01, \eta_{\mathrm{p}}{ }^{2}=.22$, and sequence, $F(1,13)=5.40, p=.03, \eta_{\mathrm{p}}{ }^{2}=.29$. The Block $\times$ Sequence interaction was not significant, $F(6,78)<1$. For the block main effect, Duncan's multiple-range test indicated withinsession performance improvements on both sequences from Blocks 3 to $9\left(\mathrm{M}_{\text {Block3 }}=520 \mathrm{~ms}\right.$ and $\mathrm{M}_{\text {Block } 9}=498 \mathrm{~ms} ; p=$ $.02)$. Interestingly, though, the main effect of sequence indicated better acquisition performance on Sequence A $(M=506$ $\mathrm{ms})$ than on Sequence B $(M=526 \mathrm{~ms})$, which reflects lower acquisition performance for the interrupted Sequence B.

Retention and intermanual transfers To account for skill learning and improved task representation following acquisition in the PP and OBS practice conditions, we analyzed the gains in performance (i.e., from pretest to posttest) for each of the retention and transfer tests (Fig. 1). Performance gains were assessed by computing the difference (in percentages) between the mean RT on every retention and transfer test block from pre- to posttest. Positive values indicate improved performance. The difference between these measures highlights practice-related performance improvements in terms of task representation and learning. Thus, we analyzed performance improvements using a Group $(\mathrm{PP}, \mathrm{OBS}) \times$ Test (retention, visual-spatial transfer, motor transfer $) \times$ Sequence $(A, B)$ ANOVA with repeated measures on the factors Test and Sequence. 

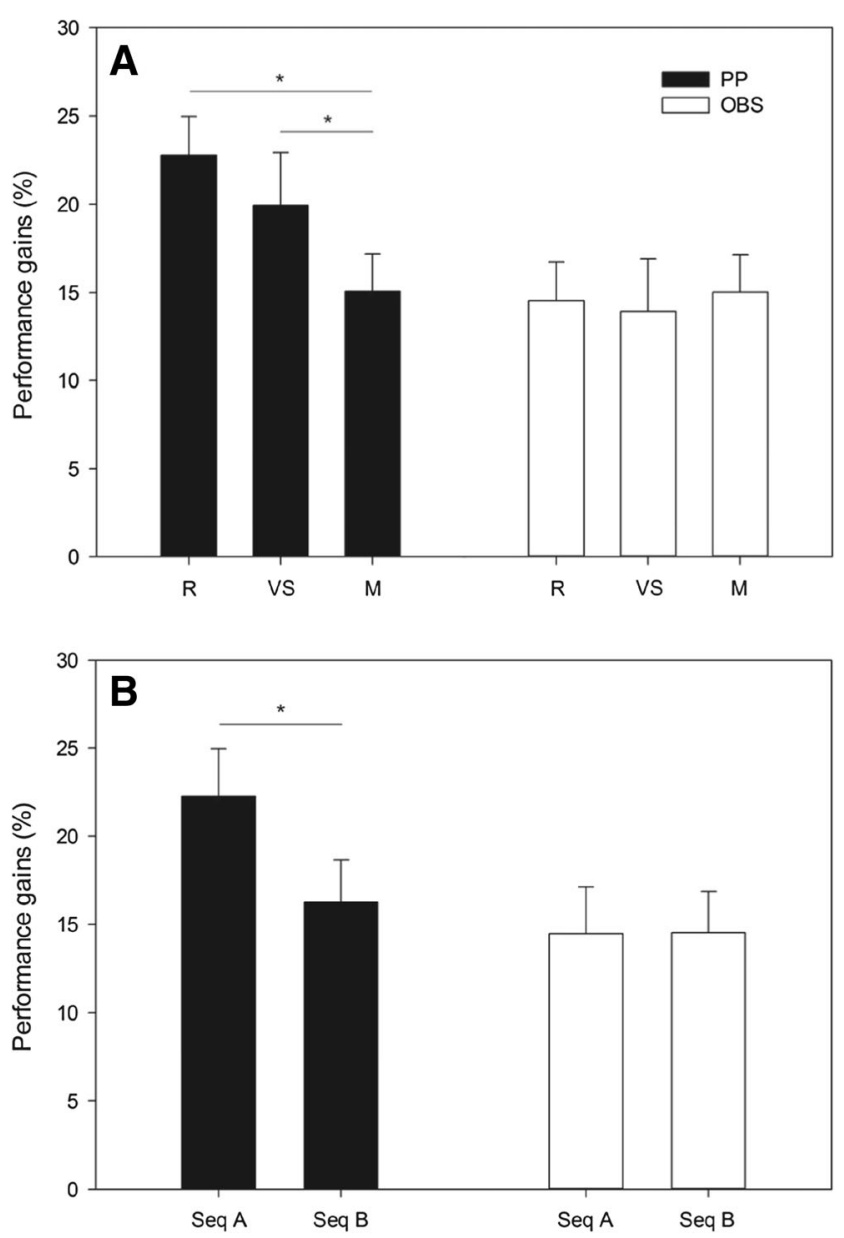

Fig. 1 (A) Mean performance gains (in percentages) from pretest to posttest for both sequences of the retention (R), visual-spatial (VS), and motor (M) transfer tests in the PP and OBS practice groups. (B) Mean performance gains (in percentages) from pretest to posttest on all tests for Sequence A (Seq A) and Sequence B (Seq B) in the PP and OBS practice groups. Error bars reflect the standard errors of the means. ${ }^{*} p<.05$

The analysis revealed main effects of group, $F(1,26)=$ $8.37, p<.01, \eta_{\mathrm{p}}{ }^{2}=.24$; test, $F(2,52)=4.50, p=.01, \eta_{\mathrm{p}}{ }^{2}=$ .15 ; and sequence, $F(1,26)=9.56, p<.01, \eta_{\mathrm{p}}{ }^{2}=.27$, as well as significant Group $\times$ Test, $F(2,52)=3.75, p=.03, \eta_{\mathrm{p}}{ }^{2}=.13$, and Group $\times$ Sequence, $F(1,26)=13.39, p<.01, \eta_{\mathrm{p}}{ }^{2}=.34$, interactions. The analysis failed to detect significant Test $\times$ Sequence, $F(2,52)=2.65, p=.08$, and Group $\times$ Test $\times$ Sequence, $F(2,52)=1.31, p>.05$, interactions. For the Group $\times$ Test interaction (see Fig. 1A), post-hoc comparisons indicated that the PP group expressed higher performance improvements on the retention and visual-spatial transfer tests $(M \mathrm{~s}=23.4 \%$ and $20.1 \%$, respectively; $p>.05)$ than on the motor-transfer test $(M=15.6 \% ; p<.001$ and $p=.02$ vs. the retention and visual-spatial tests, respectively). Interestingly, however, for the OBS group, no significant differences were found between the retention $(M=14.6 \%)$, visualspatial transfer $(M=14.5 \%)$, and motor-transfer $(M=$ $14.3 \%)$ tests. Note that the PP group showed significantly higher performance improvements than the OBS group on the retention and visual-spatial transfer tests, but not on the motor-transfer test. These findings are in accordance with previous studies showing that the visual-spatial coding system mainly governs sequence production following physical practice (see Boutin et al., 2010). Finally, further analysis of the Group $\times$ Sequence interaction revealed that the PP group expressed higher performance gains on Sequence A $(M=22.6 \%)$ than on Sequence B $(M=16.8 \%$; $p<.001)$, whereas sequence task improvements were not significantly different between Sequence A and Sequence B in the OBS group $(M \mathrm{~s}=14.2 \%$ and $14.7 \%$, respectively, $p>$ .05 ; see Fig. 1B). The latter finding highlights the fact that an observer can avoid errors through observational learning (Andrieux \& Proteau, 2014; Badets et al., 2006). It is worth noting that the PP group only showed higher performance gains than the OBS group on Sequence A $(p<.001)$. The group differences on Sequence B did not reach significance $(p>.05)$.

Sequence-specific learning To differentiate sequencelearning from generalized practice effects, we analyzed mean RT data during the posttest from the noninterrupted Sequence $A$ and a new, unpracticed sequence using a Group (PP, OBS) $\times$ Sequence (Sequence A, new sequence) factorial design with repeated measures on the last factor. The analysis revealed main effects of group, $F(1,26)=20.56, p<.001, \eta_{\mathrm{p}}{ }^{2}=.44$, and sequence, $F(1,26)=30.01, p<.001, \eta_{\mathrm{p}}{ }^{2}=.54$. The Group $\times$ Sequence interaction was not significant, $F(1,26)<1$. The main effect of group indicated that the PP group ( $M=426 \mathrm{~ms})$ outperformed the OBS group $(M=513 \mathrm{~ms})$. Most importantly, and as expected, further analysis of the sequence main effect revealed that both groups performed better on Sequence A ( $M=$ $444 \mathrm{~ms})$ than on the new sequence $(M=496 \mathrm{~ms})$, thus providing a clear indication of sequence-specific learning in both groups.

\section{Accuracy: error data analysis}

Pretest As for the RT data analysis, we ran a Group (PP, OBS) $\times$ Test (retention, visual-spatial transfer, motor transfer) $\times$ Sequence (A, B) ANOVA with repeated measures on the factors Test and Sequence. The analysis revealed a significant main effect of test, $F(2,52)=11.68, p<.001, \eta_{p}^{2}=.31$, with fewer errors produced on the retention test ( $M=2.3$ errors) than on the visual-spatial $(M=4.2$ errors; $p<.001)$ and motortransfer $(M=4.5 ; p<.001)$ tests, which did not differ $(p>.05)$. The main effects of group, $F(1,26)=1.59$, and sequence, $F(1$, 26) $<1$, as well as the Group $\times$ Test, $F(2,52)<1$, Group $\times$ Sequence, $F(1,26)<1$, Test $\times$ Sequence, $F(2,52)=2.63$, and Group $\times$ Test $\times$ Sequence, $F(2,52)<1$, interactions, were not significant, $p \mathrm{~s}>.05$. 
Acquisition This analysis was conducted using only the data from the PP group. Again, we separately analyzed (i) the noninterrupted acquisition blocks during early practice, using a Block (1-2) × Sequence (A, B) ANOVA with repeated measures on both factors, and (ii) the following seven practice blocks, using a Block (3-9) $\times$ Sequence (A, B) factorial design, also with repeated measures on both factors. The first analysis failed to detect main effects of group, $F(1,13)=3.21$, and sequence, $F(1,13)=2.86$, or a Block $\times$ Sequence interaction, $F(1,13)<1$. The second analysis revealed main effects of block, $F(6,78)=10.17, p<.001, \eta_{\mathrm{p}}{ }^{2}=.44$, and sequence, $F(1,13)=8.01, p=.01, \eta_{\mathrm{p}}{ }^{2}=.38$. The Block $\times$ Sequence interaction was not significant, $F(6,78)=1.24, p>.05$. For the block main effect, Duncan's multiple-range test indicated within-session performance improvements in accuracy on both sequences from Blocks 3 to $9\left(M_{\text {Block } 3}=3.9\right.$ errors and $\left.M_{\text {Block } 9}=1.3 ; p<.001\right)$. The main effect of sequence indicated higher error rates on Sequence A ( $M=3.1$ errors) than on Sequence B $(M=2.3)$ during acquisition, likely explained by a probabilistic lower rate of erroneous keypresses during the practice of the interrupted Sequence B.

Retention and intermanual transfers To account for improved task accuracy following acquisition in the PP and OBS practice conditions, we analyzed gains in accuracy (i.e., from pretest to posttest) for each of the retention and transfer tests. Error reductions or gains in accuracy were assessed by computing the difference (in percentage) between the mean error data on every retention and transfer test block from pre- to posttest. Positive values indicated improved performance accuracy. Note that in the absence of error production during the pretest, no gain value was assigned to the participant (less than $10 \%$ of missing values in both groups). Thus, we analyzed accuracy improvements using a Group (PP, OBS) $\times$ Test (retention, visual-spatial transfer, motor transfer) $\times$ Sequence $(\mathrm{A}, \mathrm{B})$ ANOVA with repeated measures on the factors Test and Sequence.

The analysis revealed a significant Group $\times$ Test $\times$ Sequence interaction, $F(2,30)=3.80, p=.03, \eta_{\mathrm{p}}{ }^{2}=.20$. The analysis failed to detect a significant main effect of group, $F(1,15)=1.14$; test, $F(2,30)=1.03$; or sequence, $F(1,15)<$ 1 , or a Group $\times$ Test, $F(2,30)=2.70$; Group $\times$ Sequence, $F(1$, $15)=1.03$; or Test $\times$ Sequence, $F(2,30)=1.49$, interaction, $p \mathrm{~s}$ $>$.05. Post-hoc comparisons indicated decreased performance accuracy on Sequence B for the PP group during the retention test $(M=-186.7 \%)$ than on Sequence A $(M=20.6 \%$ improvements; $p<.01)$, whereas no differences were evident on the visual-spatial $\left(M S E_{\mathrm{qA}}=-47.5 \%\right.$ and $M S E_{\mathrm{qB}}=-15.3 \% ; p>$ $.05)$ and motor-transfer $\left(M S E_{\mathrm{qA}}=-39.6 \%\right.$ and $M S E_{\mathrm{qB}}=-$ $22.6 \% ; p>.05)$ tests. In the OBS group, however, no significant differences were found between Sequences $A$ and $\mathrm{B}$ on the retention $\left(M S E_{\mathrm{qA}}=-13.72 \%\right.$ and $M S E_{\mathrm{qB}}=$ $28.3 \% ; p>.05)$, visual-spatial transfer $\left(M S E_{\mathrm{qA}}=-31.2 \%\right.$ and $\left.M S E_{\mathrm{qB}}=-74.4 \% ; p>.05\right)$, and motor-transfer $\left(M S E_{\mathrm{qA}}=\right.$ $-1.2 \%$ and $\left.M S E_{\mathrm{qB}}=23.6 \% ; p>.05\right)$ tests. The present findings indicate that, as opposed to the OBS group, for the PP group, the interruption of Sequence B did affect the learning and accurate expression of the interrupted motor sequence at later recall during the postacquisition retention test.

Sequence-specific learning We analyzed the mean error data during posttest from the noninterrupted Sequence A and a new, unpracticed sequence using a Group (PP, OBS) $\times$ Sequence (Sequence A, new sequence) factorial design with repeated measures on the last factor. The analysis revealed a main effect of sequence, $F(1,26)=8.72, p<.01, \eta_{\mathrm{p}}{ }^{2}=.25$, with higher accuracy during production of Sequence A $(M=$ 2.8 errors) than on the new, unpracticed sequence $(M=4.4$ errors; $p<.01$ ), which provides an additional indication of sequence-specific learning in both groups. The main effect of group, $F(1,26)=2.46$, and the Group $\times$ Sequence interaction, $F(1,26)<1$, were not significant, $p s<.05$.

\section{Discussion}

The main purpose of this study was to assess two observationallearning alternative hypotheses by using an original, interrupted motor-learning task paradigm. From the AON perspective, it is suggested that action observation and physical practice share common action representation networks during skill learning. According to this account, if the performance of the physical group is hampered by motor-task interruption, we could then expect a comparable weakening of skill performance for observers. Conversely, if an observer can avoid detected errors during learning, then such motor-task interruption should not hinder skill learning (Andrieux \& Proteau, 2014; Badets et al., 2006). Clearly, the present results support the second hypothesis. Findings during the posttest phase for both speed and accuracy measures revealed that the learning of the interrupted motor sequence was hampered only in the physical practice group. For observers, however, no difference was apparent between the two learned motor sequences.

To interpret this last finding, we suggest that, in contrast to practitioners who have built an action representation from the motor and perceptual mechanisms during the acquisition phase, observers have built a more abstract representation of the task from a single perceptual mechanism (Badets \& Blandin, 2010). From a theoretical point of view, such an abstract representation developed following an action observation accords with the associative sequence-learning hypothesis, which suggests that the link between sensory and motor features is learning-dependent and likewise malleable (Cook, Bird, Catmur, Press, \& Heyes, 2014; Heyes, 2001). Indeed, in contrast to an innate neuronal network that affords exact sharing between observation and physical practice (e.g., the 
AON), a plastic associative mechanism permitted equal learning of both tasks through an observation condition.

Importantly, the results of the physical practice group during the posttest revealed that the learned sequence is based more on a perceptual than on a motor mechanism, as indexed by better performance on both the retention and visual-spatial transfer tests than on the motor-transfer test. Consequently, for observers, this learning strategy from a visual-spatial code facilitated the construction of an abstract representation of the task, which yielded effective and equivalent learning of both the interrupted and noninterrupted sequences. This interpretation accords with Andrieux and Proteau (2014), who suggested that observers might be able to code relevant information from a wide performance spectrum (i.e., from an imperfect/novice model to a near-perfect/expert model). In the present study, observers were able to learn the two different sequences at the same level by constantly using a safety strategy that prevented coding of the interrupted features of the task (as the physical practice group did). This safety strategy is also apparent during the posttest performance, in which the observers did not show any change in speed and accuracy performance between the retention, visual-spatial, and motor tests, in contrast to the physical practice group.

However, another interpretation could arise from the possibility that the interrupted task benefited skill learning for the observers. Indeed, this alternative perspective is related to the well-known "Zeigarnik effect" in the (cognitive) psychology domain, which reveals better recall performance after learning of a series of different actions (like drawing a vase) that have been interrupted than for noninterrupted actions (Zeigarnik, 1927; see Seifert \& Patalano, 1991, for experimental factors that favor or do not favor this effect). Hence, the interruption of a motor sequence in the present study might have facilitated its acquisition through action observation by way of goal tension activation on the interrupted motor sequence (see Lewin, 1961; Mäntylä \& Sgaramella, 1997, for this interpretation regarding goal activation). This interpretation has been used in the DMI literature with a similar effect based on motor performance, which is called "the intention superiority effect" (Badets et al., 2006). The intention superiority effect states that the different actions for to-be-enacted behaviors are stored in a privileged and/or heightened level of activation in memory until the intention is satisfied by an overt behavior. Adapting this idea to the present paradigm, we would argue that for observers who have learned the complete motor sequence during the first and second blocks of acquisition, the interrupted motor sequence on the following blocks generated an additional activation for attaining the main goal, which was the total completion of the motor sequence. In turn, the evoked additional activation may have allowed equivalent learning of the noninterrupted motor sequence.

Finally, it is important to note that both of these theoretical interpretations are suitable for observational learning but not for physical practice learning. Indeed, our findings revealed different motor-learning outcomes between the physical and observational practice groups, which emphasizes that both practice conditions rely on specific cognitive mechanisms (Badets \& Blandin, 2010; Badets et al., 2006; Hayes et al., 2012; Shea et al., 2000). In the physical practice group, participants learn the task from a motor condition, which precludes them from avoiding task interruption processing and the elaboration of an optimal motor-sequence representation. In contrast, the perceptual condition allowed observers to adopt a safety strategy to develop and preserve efficient representation of the interrupted motor sequence.

To conclude, we would emphasize that the present study is the first direct demonstration that observers are able to develop a safety mechanism that allows learning a motor sequence without suffering from the deficiency of a model's performance. Indeed, previous studies had revealed that observers were able to use efficient strategies from a wide spectrum of model performances (Andrieux \& Proteau, 2014), or from a feedback schedule (Badets \& Blandin, 2010), but our findings go beyond these statements by highlighting that an interrupted observed motor task can be learned without performance loss.

\section{References}

Andrieux, M., \& Proteau, L. (2013). Observation learning of a motor task: Who and when? Experimental Brain Research, 229, 125-137.

Andrieux, M., \& Proteau, L. (2014). Mixed observation favors motor learning through better estimation of the model's performance. Experimental Brain Research, 232, 3121-3132.

Badets, A., \& Blandin, Y. (2010). Feedback schedules for motor-skill learning: The similarities and differences between physical and observational practice. Journal of Motor Behavior, 42, 257-268.

Badets, A., Blandin, Y., Bouquet, C., \& Shea, C. H. (2006). The intention superiority effect in motor-skill performance. Journal of Experimental Psychology: Learning, Memory, and Cognition, 32, 491-505.

Badets, A., \& Osiurak, F. (2015). A goal-based mechanism for delayed motor intention: Considerations from motor skills, tool use and action memory. Psychological Research, 79, 345-360.

Behne, T., Carpenter, M., Call, J., \& Tomasello, M. (2005). Unwilling versus unable: Infants' understanding of intentional action. Developmental Psychology, 41, 328-337.

Boutin, A., Fries, U., Panzer, S., Shea, C. H., \& Blandin, Y. (2010). Role of action observation and action in sequence learning and coding. Acta Psychologica, 135, 240-251.

Buchanan, J. J. (2015). Perceptual estimates of motor skill proficiency are constrained by the stability of coordination patterns. Journal of Motor Behavior, 47, 453-464.

Cohen, J. (1988). Statistical power analysis for the behavioral sciences (2nd ed.). Hillsdale, NJ: Erlbaum.

Cook, R., Bird, G., Catmur, C., Press, C., \& Heyes, C. M. (2014). Mirror neurons: From origin to function. Behavioral and Brain Sciences, 37, 177-241.

Franceschini, M., Agosti, M., Cantagallo, A., Sale, P., Mancuso, M., \& Buccino, G. (2010). Mirror neurons: Action observation treatment 
as a tool in stroke rehabilitation. European Journal of Physical and Rehabilitation Medicine, 46, 517-523.

Hayes, S. J., Elliott, D., Andrew, M., Roberts, J. W., \& Bennett, S. J. (2012). Dissociable contributions of motor-execution and actionobservation to intramanual transfer. Experimental Brain Research, $221,459-466$.

Heyes, C. (2001). Causes and consequences of imitation. Trends in Cognitive Sciences, 5, 253-261. doi:10.1016/S1364-6613(00)01661-2

Hodges, N. J., Williams, A. M., Hayes, S. J., \& Breslin, G. (2007). What is modelled during observational learning? Journal of Sports Sciences, 25, 531-545.

Kleiner, M., Brainard, D., \& Pelli, D. (2007). What's new in Psychtoolbox3? Perception, 36(ECVP Abstract Suppl), 14.

Lewin, K. (1961). Intention, will, and need. In T. Shipley (Ed. \& Trans.), Classics in psychology (pp. 1234-1288). New York, NY: Philosophical Library.

Loucks, J., \& Meltzoff, A. N. (2013). Goals influence memory and imitation for dynamic human action in 36-month-old children. Scandinavian Journal of Psychology, 54, 41-50.

Mäntylä, T., \& Sgaramella, T. (1997). Interrupting intentions: Zeigarniklike effects in prospective memory. Psychological Research, 60, 192-199.

Maslovat, D., Hodges, N. J., Krigolson, O. E., \& Handy, T. C. (2010). Observational practice benefits are limited to perceptual improvements in the acquisition of a novel coordination skill. Experimental Brain Research, 204, 119-130.

Meltzoff, A. N. (1995). Understanding the intentions of others: Re-enactment of intended acts by 18-month-old children. Developmental Psychology, $31,838-850$.
Oldfield, R. C. (1971). The assessment and analysis of handedness: The Edinburgh inventory. Neuropsychologia, 9, 97-113. doi:10.1016/ 0028-3932(71)90067-4

Press, C. (2011). Action observation and robotic agents: Learning and anthropomorphism. Neuroscience and Biobehavioral Reviews, 35, $1410-1418$.

Rohbanfard, H., \& Proteau, L. (2011). Learning through observation: A combination of expert and novice models favors learning. Experimental Brain Research, 215, 183-197.

Seifert, C. M., \& Patalano, A. L. (1991, July). Memory for incomplete tasks: A re-examination of the Zeigarnik effect. Paper presented at the Thirteenth Annual Conference of the Cognitive Science Society, Chicago, IL.

Shea, C. H., Wright, D. L., Wulf, G., \& Whitacre, C. (2000). Physical and observational practice afford unique learning opportunities. Journal of Motor Behavior, 32, 27-36.

Subiaul, F., Patterson, E. M., \& Barr, R. (2016). The cognitive structure of goal emulation during the preschool years. British Journal of Developmental Psychology, 34, 132-149.

Thomas, R., Sink, J., \& Haggard, P. (2013). Sensory effects of action observation: Evidence for perceptual enhancement driven by sensory rather than motor simulation. Experimental Psychology, 60, 335346.

Whiten, A., \& Ham, R. (1992). On the nature and evolution of imitation in the animal kingdom: Reappraisal of a century of research. Advances in the Study of Behaviour, 11, 239-283.

Zeigarnik, B. (1927). Uber das Behalten von erledigten und unerledigten Handlungen [On the retention of completed and uncompleted transactions]. Psychologische Forschung, 9, 1-85. 